\title{
Papel de las pruebas rápidas (POCT) en el diagnóstico del SARS-COV-2, agente causal de COVID-19
}

\author{
Role of Rapid Tests (POCT) in the Diagnosis of SARS-VOC-2, Causal Agent \\ of COVID-19
}

Carmen Cecilia Almonacid Urrego ${ }^{1}$, María Vilma Giratá Pedraza², Irlena Salcedo Pretelt ${ }^{3}$, Isabel Cristina Almonacid Urrego ${ }^{4}$

\section{Resumen}

El estándar de oro actual para la detección de SARS-CoV-2, agente causal de la pandemia de neumonía atípica (COVID-19) que apareció por primera vez en la ciudad de Wuhan (provincia de Hubei, China) en diciembre de 2019 (1), es la RT-qPCR. El protocolo estándar implica la transcripción inversa de ARN de SARS-CoV-2 en cadenas de ADN complementarias $(\mathrm{ADNc})$, seguida de la amplificación de regiones específicas del ADNc. Este procedimiento demanda varias horas para ser completado y deriva en que la información final del estado de la infección pueda demorar hasta 24 horas. Ante la necesidad de disminuir el riesgo de una posible propagación viral dentro de la población originada por la rápida transmisión del SARS-CoV-2, se ha buscado prevenir el contagio, la propagación nosocomial y la transmisión comunitaria posterior, a través de la identificación rápida de casos sospechosos, y predecir las posteriores ondas infecciosas de recurrencia viral. Para esto, se vienen desarrollando métodos de laboratorio rápidos o point of care testing (POCT), que disminuyen el tiempo de diagnóstico y minimizan el riesgo de contagio por parte de los operadores.

\footnotetext{
1. Ph. D. Biomedicina, M. Sc. Microbiología, con énfasis en Bioquímica, Bacterióloga y laboratorista clínico. Grupo ECZA, Facultad de Ciencias de la Salud, Universidad Colegio Mayor de Cundinamarca, Colombia. Asociación Científica Latina (ASCILA). ORCID: https://orcid.org/0000-0002-0218-9367

2. Esp. bacterióloga y laboratorista clínica, y especialista en Administración Hospitalaria. Grupo ECZA, Facultad de Ciencias de la Salud, Universidad Colegio Mayor de Cundinamarca, Colombia. Asociación Científica Latina (ASCILA).

ORCID: https://orcid.org/0000-0001-7375-7926

3. Mg. en Salud Pública, auditora de calidad en salud, bacterióloga y laboratorista clínica. Secretaría Distrital de Salud de Bogotá, Grupo ECZA, Facultad de Ciencias de la Salud, Universidad Colegio Mayor de Cundinamarca.

ORCID: https://orcid.org/0000-0002-9864-5426

4 Mg. Oncología Molecular, Mg. Ginecología Oncológica, M. D. cirujano especialista en Patología. Hospital Central de la Policía, Laboratorio de Salud Pública de Cundinamarca, Grupo ECZA, Facultad de Ciencias de la Salud, Universidad Colegio Mayor de Cundinamarca.

ORCID: https://orcid.org/0000-0002-0218-9367
}

Correo electrónico de correspondencia: calmonacidu@unicolmayor.edu.co

Institución donde se realizó el trabajo: Universidad Colegio Mayor de Cundinamarca 
Palabras claves: SARS-CoV-2, coronavirus humano, Covid 19, POCT.

\section{Abstract}

The gold test to detect SARS-CoV-2, the etiologic agent that leads to the pandemic of atypical pneumonia (COVID 2019) that first appeared in Wuhan City, Hubei Province of China in December 2019 (1), is the RT-qPCR. The standard protocol involves reverse transcription of SARS-CoV-2 RNA into complementary DNA strands (cDNA), followed by the amplification of cDNA specific regions, a procedure that takes several hours to complete and which results in the final information from the infection status can take up to 24 hours. For this reason, and due to the need to reduce the risk of possible viral spread within the population caused by the fast transmission of SARS-CoV-2, in order to prevent nosocomial spread and subsequent community transmission through the quick identification of suspected cases, and to predict the further infectious waves of viral recurrence, rapid laboratory methods or Point of Care Testing (POCT) are being developed to reduce the diagnosis time and minimize the risk of contagion by the operators. These tests are discussed below.

Keywords: Human coronavirus; SARS-CoV-2; COVID-19; POCT.

Metodologías POCT para el estudio de SARS-CoV-2 (2)

\section{Serológicas}

Estas pruebas detectan, en sangre, suero o plasma $(2,3)$, los anticuerpos generados como respuesta a la infección por SARSCoV-2 $(4,5,6)$. En pacientes jóvenes y sanos, la IgM y la IgA aparecen entre el cuarto y el sexto día del inicio de los síntomas, con pico en el día $14(7,8)$. En pacientes inmunodeprimidos y crónicos la respues- ta se da entre el séptimo y decimocuarto día. Por su parte, los anticuerpos IgG se retrasan en algunos casos y aparecen entre los días 15 y $21(8,9,10,11)$, de modo que aparece su pico entre los días 17 y 19. Por ello, una POCT serológica realizada demasiado temprano puede no caracterizar adecuadamente el estado de los pacientes y generar falsos negativos $(7,12,13,14,15)$.

Las metodologías usadas son Elisa e inmunocromatografía de flujo lateral con partículas de oro coloidal (16). Las proteínas 
virales de la espiga (S) y la nucleocápside (N) actúan como antígenos. Otros formatos, como el de inmunoflorescencia y búsqueda de anticuerpos neutralizantes, solo están disponibles en laboratorios de referencia o investigación (17). La sensibilidad de los productos comerciales varía entre el $40 \%$ y $93 \%$, y la especificidad entre $93 \%$ y $100 \%(5,8,10,12,18)$, por lo que la tasa de casos falsos negativos puede ser sustancial (8).

Asimismo, se ha reportado reactividad cruzada por la inmunidad adquirida contra otros coronavirus como el SARS-CoV $(4,19,20)$. Por ello, la Organización Panamericana de la Salud (OPS) y la Organización Mundial de la Salud (OMS) recomiendan validar y evaluar el rendimiento de estas pruebas en términos de especificidad y sensibilidad, y han aconsejado que cada país realice sus propias validaciones antes de implementarlas $(20,21)$. Igualmente, aunque estas pruebas no las indican para el diagnóstico clínico temprano, las consideran herramientas valiosas para la vigilancia, predicción del resultado de la enfermedad y la investigación epidemiológica $(19,20,21)$. La lista de los ensayos en evaluación está disponible en el sitio web de la Foundation for Innovative New Diagnostics (FIND) (22).

Para mejorar los criterios de calidad y el índice de detección se ha propuesto emplear la combinación rtRT-PCR e IgM/IgG en pacientes que seroconvierten $(23,24)$; obtener muestras de suero emparejadas (en la fase aguda y convaleciente); utilizar como antígeno secciones de la proteína $S$ que sean particularmente distintas entre los coronavirus, y recurrir a ensayos basados en la metodología de antígeno recombinante $(3,19)$.

\section{Ventajas $(6,17,25)$ :}

- Rapidez en la obtención de resultados

- Producción masiva y de bajo costo

- Baja o nula carga viral de las muestras usadas

- Adaptables a diferentes formatos de diagnóstico

- Pueden utilizarse en el sitio de la toma de la muestra

\section{Desventajas $(6,17,25)$ :}

- Posibilidad de falsos negativos o positivos

- Problemas de reproducibilidad entre lotes

- Resultado esencialmente cualitativo o semicuantitativo

- Requieren la respuesta inmune del individuo

\section{Antigénicas}

Con estas pruebas se detectan componentes estructurales del virus SARS-CoV-2 como la proteína de la nucleocápside $(\mathrm{N})$ $(26,27)$ y las subunidades S1 o S2 de la proteína espiga $(S)(28,29)$, en muestras de esputo o hisopado nasofaríngeo u orofaríngeo, que se recolectan en etapas tempranas de la enfermedad $(30,31,32,33)$. 
Las metodologías disponibles para estos ensayos son la inmunocromatografía con incorporación de partículas de oro coloidal y los ensayos inmunofluoresecentes (34). También se han desarrollado pruebas inmunocromatográficas de fluorescencia que correlacionan $100 \%$ con la qRT-PCR (27). Todas ellas utilizan anticuerpos monoclonales específicos contra el SARSCoV-2 que son complejos. Por ello, para mejorar los valores diagnósticos se requiere concentrarlos o amplificar la fase de detección (35). La lista de los ensayos en evaluación está disponible en la página de la FIND (22).

Teóricamente el antígeno viral es el marcador específico del virus y precede la aparición de anticuerpos; por lo tanto, la detección del antígeno implica replicación activa del virus e indica infección actual por SARS-CoV-2 (25). Sin embargo, aunque hay estudios que demuestran buena sensibilidad, especificidad y correlación de estas pruebas con la qRT-PCR $(27,36)$, otros la refutan y plantean la posibilidad de obtener falsos negativos $(6,21,25,30)$. De igual manera, mientras algunos laboratorios señalan que no hay reacción cruzada con otros coronavirus, otros la confirman (37), de manera que no se puede descartar la presencia de falsos positivos (6).

Todo ello ha llevado a la OMS a no recomendar, hasta ahora, el uso de pruebas rápidas antigénicas para la atención del paciente, aunque invita encarecidamente a investigar su rendimiento y posible utilidad diagnóstica (21).

\section{Ventajas $(10,24,35,38)$}

- Rapidez, sencillez y facilidad de estandarización

- Adecuada sensibilidad si se utiliza fluorimetría

- Buena correlación con la qRT-PCR

- Buen valor predictivo positivo

- No presentan problemas de contaminación

\section{Desventajas $(6,17)$}

- Riesgo biológico en la toma de la muestra

- No permiten procesar gran número de muestras en periodos cortos de tiempo

- Posibilidad de obtener falsos positivos

- Carga viral presente no cuantificable

- Al momento es incierta su utilidad diagnóstica

\section{Moleculares}

Estas muestras se fundamentan en la amplificación isotérmica mediada por bucle de transcripción inversa RT-LAMP, método que se ha venido utilizado para la detección rápida de virus ARN como SARS-CoV, MERS-CoV e influenza $(39,40,41,42)$. No obstante, aún están en desarrollo para la identificación de SARS- 
CoV-2 $(45,46,47,48,49,50)$ y no se ha avalado su utilidad en entornos clínicos (2).

Los ensayos LAMP incluyen la amplificación exponencial de secuencias específicas de ácido nucleico a una temperatura constante en la que se emplean un total de seis cebadores: dos internos, dos externos y dos de bucle, que facilitan y aceleran la amplificación y detección. Incorporan también una ADN polimerasa de desplazamiento de cadena, que actúa a una temperatura constante de 60 a $65^{\circ} \mathrm{C}(39,46,51)$. La formación en estructura de bucle elimina el paso de desnaturalización a $90{ }^{\circ} \mathrm{C}$ que es esencial en la qRT-PCR para obtener ADN monocatenario $(52,53)$.

\section{Ventajas}

- Mayor velocidad de amplificación $(45,51,54)$

- Especificidad y sensibilidad comparables a la qRT-PCR (46)

- Se obtiene más ADN que en la qRTPCR $(51,55,56,57)$

- Permiten muestras clínicas variables (hisopados orales, de garganta, nasales o nasofaríngeos y lavado broncoalveloar) (2)

\section{Desventajas $(51,58)$}

- Evitan la inclusión de un control interno de inhibición de la PCR

- Requieren de un sistema de diseño de cebador complejo
- El tamaño del producto final limita aplicaciones posteriores como la clonación

- Durante las manipulaciones del ensayo son sensibles a los contaminantes en aerosol

Otras POCT moleculares en ensayo son la metodología Sherlock $(4,59,60)$ y un sistema de detección basado en Cas13a (61). ¿Son útiles o no estas pruebas? Depende de su correcta aplicación (figura 1) e interpretación clínica (tabla 1) 
Figura 1. Uso de las pruebas POCT en COVID-19.

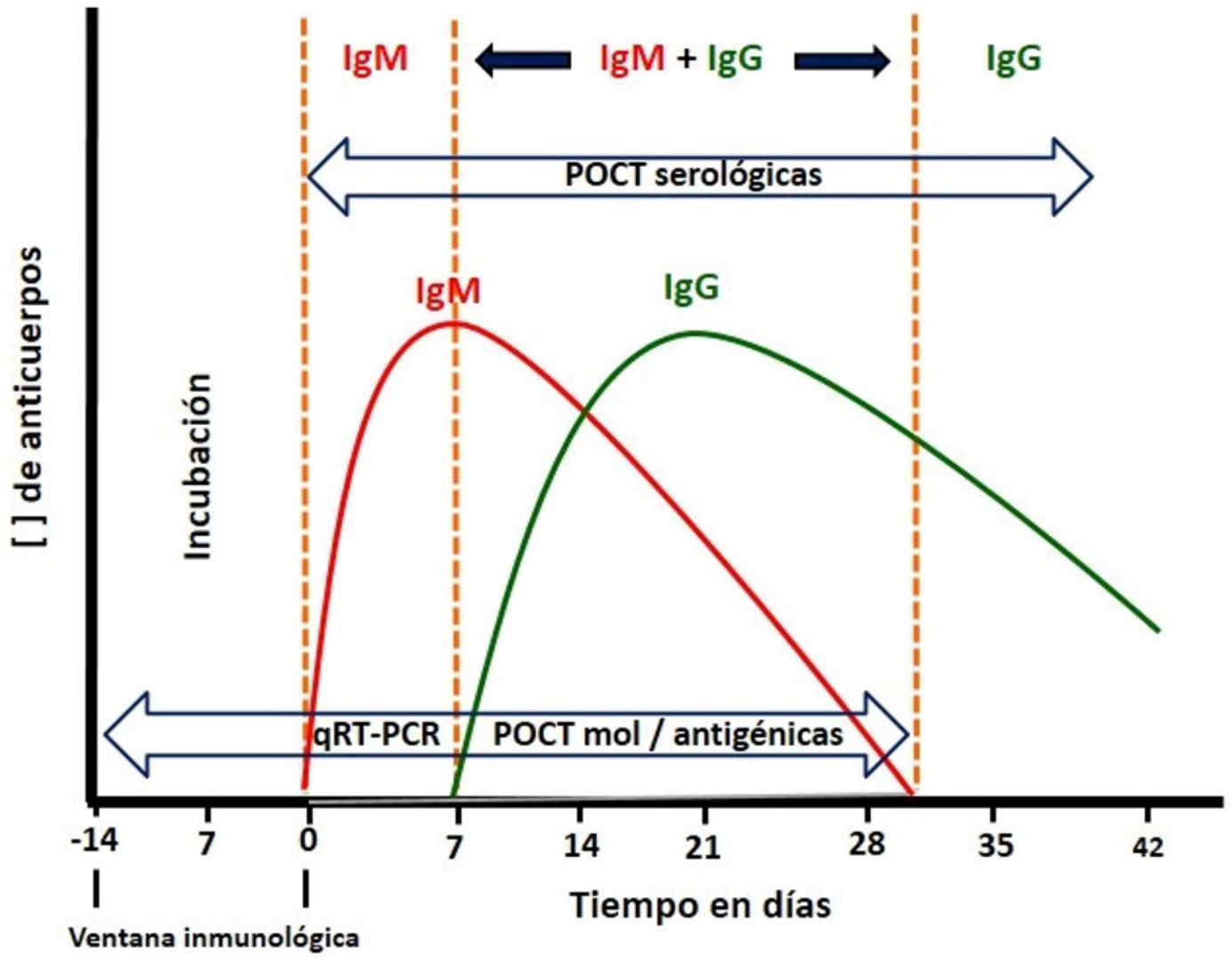

Tabla 1. Interpretación clínica pruebas POCT (62).

\begin{tabular}{|c|c|c|c|}
\hline \multicolumn{3}{|c|}{ Resultado } & \multirow{2}{*}{ Significado clínico } \\
\hline IgM & IgG & qRT-PCR & Negativo \\
\hline- & - & - & Positivo \\
\hline- & - & + & Probable positivo \\
\hline+ & - & & Probable positivo \\
\hline+ & + & & Positivo \\
\hline- & + & - & Recuperado. Infección resuelta \\
\hline- & + & + & \\
\hline
\end{tabular}




\section{Referencias}

1. Zhu, N, Zhang D, Wang W, Li X, Yang B, Zhao $\mathrm{X}$, et al. A Novel Coronavirus from Patients with Pneumonia in China, 2019. N Engl J Med. 2020 Feb 20; 382(8):727-733.

2. Younes $\mathrm{N}, \mathrm{Al}$-Sadeq D, Al-Jighefee $\mathrm{H}$, Younes $\mathrm{S}$, Al-Jamal O, Daas $\mathrm{H}$, et al. Challenges in Laboratory Diagnosis of the Novel Coronavirus SARS-CoV-2. Viruses. 2020 May 26; 12(6): E582.

3. Amanat F, Stadlbauer D, Strohmeier S, Nguyen T, Chromikova V, McMahon M, et al. A serological assay to detect SARS-CoV-2 seroconversion in humans. Nat Med. 2020 May 12; 10: 10.1038/ s41591-020-0913-5.

4. Udugama B, Kadhiresan P, Kozlowski H, Malekjahani A, Osborne M, Li V, et al. Diagnosing COVID-19: The Disease and Tools for Detection. ACS Nano. 2020 Apr 28; 14(4): 3822-3835.

5. Mallapaty S. Will antibody tests for the coronavirus really change everything?. Nature. 2020 Apr; 580(7805): 571-572.

6. Onoda M, Martínez Chamorro M. Pruebas diagnósticas de laboratorio de COVID-19. Asociación Española de Pediatría de Atención Primaria, Grupo de Patología Infecciosa; 2020.

7. Long Q, Liu B, Deng H, Gu GC, Deng K, Chen $\mathrm{Y}$, et al. Antibody responses to SARS-CoV-2 in patients with COVID-19. Nat Med. 2020 Jun; 26(6): 845-848.

8. Riccò M, Ferraro P, Gualerzi G, Ranzieri S, Henry B, Said Y, et al. Point-of-Care Diagnostic Tests for Detecting SARS-CoV-2 Antibodies: A Systematic Review and Meta-Analysis of Real-World Data. J Clin Med. 2020 May 18; 9(5): 1515.

9. Liu W, Liu L, Kou G, Kou G, Zheng Y, Ding Y, et al. Evaluation of Nucleocapsid and Spike Protein-based ELISAs for detecting antibodies against SARSCoV-2. J Clin Microbiol. 2020 Mar 30; 58(6): e00461-20.
10. Li Z, Yi Y, Luo X, Xiong N, Liu Y, Li S, et al. Development and clinical application of a rapid $\operatorname{IgM}-\operatorname{IgG}$ combined antibody test for SARS-CoV-2 infection diagnosis. J Med Virol. 2020 Feb 27; 10: 1002/ jmv. 25727.

11. Lippi G, Mattiuzzi C, Bovo C, Plebani M. Current laboratory diagnostics of coronavirus disease 2019 (COVID-19). Acta Biomed. 2020 May 11; 91(2): 137-145.

12. Lassaunière R, Frische A, Harboe ZB, Nielsen AC, Fomsgaard A, Krogfelt KA, et al. Evaluation of Nine Commercial SARS-CoV-2 Immunoassays. medRxiv. 2020.

13. Adams E, Ainsworth M, Anand R, Andersson M, Auckland K, Baillie K, et al. Evaluation of Antibody Testing for SARS-CoV-2 Using ELISA and Lateral Flow Immunoassays. medRxiv. 2020.

14. Imai K, Tabata S, Ikeda M, Noguchi S, Kitagawa $Y$, Matsuoka M, et al. Clinical Evaluation of an Immunochromatographic IgM/IgG Antibody Assay and Chest Computed Tomography for the Diagnosis of COVID-19. Journal of clinical virology. 2020 July; $128: 104393$.

15. Pan Y, Li X, Yang G, Fan J, Tang Y, Zhao J, et al. Serological Immunochromatographic Approach in Diagnosis with SARS-CoV-2 Infected COVID-19 Patients. J. Infect. 2020 Jul; 81: e28-e32.

16. Aviles E, Barba C, Calle D, Guamán L, López V, Saenz K, et al. Protocolo de uso de pruebas rápidas para detección de anticuerpos contra SARS-CoV-2/ COVID-19. Informe Técnico. Quito: Ministerio de Salud del Ecuador; 2020.

17. Comité Consultivo de Microbiologia Clínica- SOCHINF. Diagnóstico Microbiológico de SARSCoV-2 (COVID-19) versión 1.0. Documento de revisión. Santiago de Chile : Sociedad Chilena de Infectología (SOCHINF); 2020.

18. Döhla M, Boesecke C, Schulte B, Diegmann C, Sib E, Richter E, et al. Rapid point-of-care testing for SARS-CoV-2 in a community screening setting 
shows low sensitivity. Public Health. 2020 May; 182: 170-172.

19. Yan Y, Chang L, Wang L. Laboratory testing of SARS-CoV, MERS-CoV, and SARS-CoV-2 (2019$\mathrm{nCoV})$ : Current status, challenges, and countermeasures. Rev Med Virol. 2020 May; 30(3): e2106.

20. Organización Panamericana de la Salud. Directrices de Laboratorio para la Detección y el Diagnóstico de la Infección con el Virus COVID-19. ; 2020.

21. World Health Organization. Advice on the use of point-of-care immunodiagnostic tests for COVID-19. Scientific brief; 2020.

22. Foundation for Innovative New Diagnostics. FIND Evaluation update: SARS-CoV-2 Immunoassays. [Online]. 2020 [cited 2020 Junio 19]. Disponible en: https://www.finddx.org/covid-19/sarscov2-eval-immuno/

23. Zhou F, Yu T, Du R, Fan G, Liu Y, Liu Z, et al. Clinical course and risk factors for mortality of adult inpatients with COVID-19 in Wuhan, China: a retrospective cohort study. Lancet. 2020 Mar 28; 395(10229): 1054-1062.

24. Guo L, Ren L, Yang S, Xiao M, Chang D, Yang F, et al. Profiling Early Humoral Response to Diagnose Novel Coronavirus Disease (COVID-19). Clin Infect Dis. 2020 Mar 21.

25. Sociedad Española de Enfermedades Infecciosas y Microbiología Clínica. Documento de posicionamiento de la SEIMC sobre el diagnóstico microbiólogo de COVID-19. SIMC; 2020.

26. Wu A, Peng Y, Huang B, Ding X, Wang X, Niu P, et al. Genome Composition and Divergence of the Novel Coronavirus (2019-nCoV) Originating in China. Cell Host Microbe. 2020 Mar 11; 27(3): 325-328.

27. Diao B, Wen K, Chen J, Liu Y, Yuan Z, Han C. Diagnosis of Acute Respiratory Syndrome Coronavirus 2 Infection by Detection of Nucleocapsid Protein. MedRxiv. 2020 Mar 30.

28. Sandeep K. In Vitro Diagnostic Assay for CO-
VID-19: Recent Advances and Emerging Trends. Diagnostics. 2020 Apr; 10(4): 202.

29. Wrapp D, Wang N, Corbett K, Goldsmith J, Hsieh C, Abiona O, et al. Cryo-EM structure of the 2019$\mathrm{nCoV}$ spike in the prefusion conformation. Science. 2020 Mar 13; 367(6483): 1260-1263.

30. Zou L, Ruan F, Huang M, Liang L, Huang H, Hong Z, et al. SARS-CoV-2 Viral Load in Upper Respiratory Specimens of Infected Patients. N Engl J Med. 2020 Mar 19; 382(12): 1177-1179.

31. Pan Y, Zhang D, Yang P, Poon L, Wang Q. Viral load of SARS-CoV-2 in clinical samples. Lancet Infect Dis. 2020 Apr; 20(4): 411-412.

32. To K, Tsang O, Leung W, Raymond A, Wu T, Lung $\mathrm{D}$, et al. Temporal profiles of viral load in posterior oropharyngeal saliva samples and serum antibody responses during infection by SARS-CoV-2: an observational cohort study. Lancet Infect Dis. 2020 May; 20(5): 565-574.

33. Lippi G, Simundic A, Plebani M. Potential preanalytical and analytical vulnerabilities in the laboratory diagnosis of coronavirus disease 2019 (COVID-19). Clin Chem Lab Med. 2020 Jun 25; 58(7): 1070-1076.

34. Li W, Liu L, Chen L, Shang S. Evaluation of a Commercial Colloidal Gold Assay for Detection of Influenza A and B Virus in Children's Respiratory Specimens. Fetal Pediatr Pathol. 2020 Apr; 39(2): 93-98.

35. Loeffelholz M, Tang. Laboratory diagnosis of emerging human coronavirus infections - the state of the art. Emerg Microbes Infect. 2020 Dic; 9(1): 747-756.

36. Blairon L, Wilmet A, Beukinga I, Tré-Hardy M. Implementation of rapid SARS-CoV-2 antigenic testing in a laboratory without access to molecular methods: Experiences of a general hospital. Clin Virol. 2020 May 30; 12: 104472.

37. Okba N, Müller, M, Li W, Li W, Wang C, GeurtsvanKessel C, et al. Severe Acute Respiratory Syndro- 
me Coronavirus 2-Specific Antibody Responses in Coronavirus Disease 2019 Patients [published online ahead of print, 2020 Apr 8]. Emerg Infect Dis. 2020 Apr; 26(7).

38. Zhao J, Yuan Q, Wang H, Liu W, Liao X, Su Y, et al. Antibody responses to SARS-CoV-2 in patients of novel coronavirus disease 2019. Clin Infect Dis. 2020 Mar 28;: ciaa344.

39. Lira Carmona R, De la Cruz Pérez J, Maldonado Rodríguez, A, Rojas Montes O. Sistemas de amplificación isotérmica para la detección molecular de los virus zika, dengue y chikungunya. Mens. Bioquim. 2018; 42: 92-102.

40. Poon LL, Leung CS, Tashiro M, Chan KH, Wong BW, Yuen KY, et al. Rapid detection of the severe acute respiratory syndrome (SARS) coronavirus by a loop-mediated isothermal amplification assay. Clin Chem. 2004 Jun; 50(6): 1050-1052.

41. Pyrc K, Milewska A, Potempa J. Development of loop-mediated isothermal amplification assay for detection of human coronavirus-NL63. J Virol Methods. 2011 Jul; 175(1): 133-136.

42. Mori Y, Nagamine K, Tomita N, Notomi T. Detection of loop-mediated isothermal amplification reaction by turbidity derived from magnesium pyrophosphate formation. Biochem Biophys Res Commun. 2001 Nov 23; 289(1): 150-154.

43. Shirato K, Yano T, Senba S, Akachi S, Kobayashi T, Nishinaka T, et al. Detection of Middle East respiratory syndrome coronavirus using reverse transcription loop-mediated isothermal amplification (RTLAMP). Virol J. 2014 Aug; 8(11): 139.

44. Pang J, Wang M, Ang I, Tan S, Lewis R, Chen J, et al. Potential Rapid Diagnostics, Vaccine and Therapeutics for 2019 Novel Coronavirus (2019-nCoV): A Systematic. Review. J Clin Med. 2020 Feb 26; 9(3): 623.

45. Lu R, Zhao X, Li J, Niu P, Yang B, Wu H, et al. Genomic characterisation and epidemiology of 2019 novel coronavirus: implications for virus origins and receptor binding. Lancet. 2020 Feb 22; 395(10224): 565-574.

46. Baek Y, Um J, Antigua, Park J, Kim Y, Oh S, et al. Development of a reverse transcription-loop-mediated isothermal amplification as a rapid early-detection method for novel SARS-CoV-2. Emerg Microbes Infect. 2020 Dec; 9(1): 998-1007.

47. El-Tholoth M, Bau Haim H, Song J. A Single and Two-Stage, Closed-Tube, Molecular Test for the 2019 Novel Coronavirus (COVID-19) at Home, Clinic, and Points of Entry. ChemRxiv. 2020 Feb.

48. Lamb, L, Bartolone S, Ward E, Chancellor M. Rapid Detection of Novel Coronavirus (COVID19) by Reverse Transcription-Loop-Mediated Isothermal Amplification. MedRxiv. 2020 Feb 24.

49. Zhang Y, Odiwuor N, Xiong J, Sun L, Nyarua R, Wei $\mathrm{H}$, et al. Rapid molecular detection of SARSCoV-2 (COVID-19) virus RNA using colorimetric LAMP. MedRxiv. 2020 Feb 29.

50. Cordero L, Bartolone S, Ward E, Canciller M. Detección rápida de nuevos coronavirus (COVID-19) por amplificación isotérmica mediada por bucle de transcripción inversa. medRxiv. 2020 Feb.

51. Kashir J, Yaqinuddin A. Loop mediated isothermal amplification (LAMP) assays as a rapid diagnostic for COVID-19. Med Hypotheses. 2020 Apr 25; 141: 109786.

52. Gallas-Lindemann C, Sureshkumar P, Noack M, Sotiriadou I. Loop-Mediated Isothermal Amplification: An Advanced Method for the Detection of Giardia. In Rodriguez-Morales A. Current Topics in Giardiasis.; 2017. 109.

53. Mori Y, Tomita, N, Kanda H, Notomi T. Mori, Y., Tomita, N., Kanda, H., \& Notomi, T. (2012). Novel MoleculaDiagnostic Platform for Tropical Infectious Diseases. In Rodriguez Morales A. Current Topics in Tropical Medicine.; 2012. 445.

54. Shen M, Zhou Y, Ye J, Abdullah Al-Maskri A, Kang Y Zeng S, Cai S, et al. Recent advances and pers- 
pectives of nucleic acid detection for coronavirus. J Pharm Anal. 2020 Mar; 10(2): 97-101.

55. Shirato K, Semba S, El-Kafrawy S, Hassan A, Tolah A, Takayama I, et al. Development of fluorescent reverse transcription loop-mediated isothermal amplification (RT-LAMP) using quenching probes for the detection of the Middle East respiratory syndrome coronavirus. J Virol Methods. 2018 Aug; 258: $41-48$.

56. Njiru Z. Loop-mediated isothermal amplification technology: towards point of care diagnostics. PLoS Negl Trop Dis. 2012; 6(6): e1572.

57. Huang P, Wang H, Cao Z, Jin H, Chi H, Zhao J, et al. A Rapid and Specific Assay for the Detection of MERS-CoV. Front Microbiol. 2018 May 29; 9: 1101.

58. Lee S, Baek Y, Kim Y, Choi Y, Song M, Ahn J. OnePot Reverse Transcriptional Loop-Mediated Isothermal Amplification (RT-LAMP) for Detecting MERS-CoV. Front Microbiol. 2017 Jan 9; 7: 2166.

59. Joung J, Ladha A, Saito M, Segel M, Bruneau R, Huang $M$, et al. Point-of-care testing for COVID-19 using SHERLOCK diagnostics. MedRxiv. 2020 May 8;: 2020.05.04.20091231.

60. Wang X, Zhong M, Liu Y, Ma P, Dang L, Meng $\mathrm{Q}$, et al. Rapid and Sensitive Detection of COVID-19 Using CRISPR/Cas12a-based Detection with Naked Eye Readout, CRISPR/Cas12a-NER. Sci Bull (Beijing). 2020 May 5;: 2020;10.1016/j. scib.2020.04.041.

61. Hou T, Zeng W, Yang M, Chen W, Ren L, Ai J, et al. Development and Evaluation of a CRISPR-Based Diagnostic For 2019-Novel Coronavirus. medRxiv. 2020 Feb 25.

62. Gallegos S, Mojica J, Meza M, Cuellar C. Lineamientos para el uso de pruebas diagnósticas de SARS-COV-2 (COVID-19) en Colombia. Informe técnico. Bogotá D.C: Ministerio de Salud y Protección Social; 2020. 